\title{
Improvement of Spatial Resolution in Z Direction with Improved Energy Spread Measured using Aberration Corrected STEM with Cold Field Emission Gun
}

\author{
Hiroki Hashiguchi $^{1 *}$, Ryusuke Sagawa ${ }^{1}$, Noriaki Endo ${ }^{1}$, Eiji Okunishi ${ }^{1}$ and Yukihito Kondo ${ }^{1}$ \\ 1. JEOL Ltd. 3-1-2 Musashino Akishima Tokyo, Japan \\ * Corresponding author: hhashigu@jeol.co.jp
}

Recently, the spatial resolution of scanning transmission microscope (STEM) has dramatically improved owing to an aberration corrector. The primary benefit of the aberration corrector is a wide flat area of the Ronchigram and a large convergence angle for a STEM probe. In the STEM, the spatial resolution improves as the convergence angle increases, because diffraction limit $(\mathrm{d})$ decreases $(\mathrm{d}=0.6 \lambda / \alpha, \lambda$ : wavelength, $\alpha$ : convergence angle). In a recent aberration corrector, the allowable convergence angle has become $70 \mathrm{mrad}$ or more, since the fifth order aberration can be corrected [1]. As a result, with the corrector, atomic resolution has become realized in both image observation and analysis by EDS and/or EELS. However, the acquired STEM image is basically two-dimensional map. Although it is convenient if it is an ideal sample like mono-atomic layered sample, the practical sample, which we observe and analyze, actually contains many defects and impurities, which distribute three dimensionally. Thus, it is important to characterize the sample three dimensionally. Therefore, the three dimensional spatial resolution is necessary for the observation and analysis.

Modern aberration corrector improves depth resolution $\left(\mathrm{d}_{\mathrm{z}}\right)$ further, since larger convergence angle is obtainable due to corrections of higher order geometrical aberrations. This leads that optical slicing of the sample observation may be doable similar to confocal optical microscopy with modern aberration correctors. In this paper, we report $\mathrm{d}_{\mathrm{z}}$ obtained through various practical experimental tests. The depth resolution [2] is represented by the relation:

$$
d_{z}=\lambda / \alpha^{2}-(1)
$$

First, we examined this equation. To estimate $d_{z}$, through-focal series of the STEM images were acquired using a sample of mono-layer graphene. For this experiment, we used a $200-\mathrm{kV}$ aberration corrected microscope (JEOL: NEOARM), equipped with cold field emission gun and electron energy spectrometer (EELS). The acceleration voltage for this experiment was $80 \mathrm{kV}$ and number of images in a series was 40. Then, we calculated the standard deviation ( $\sigma$ image) of the intensities at the pixels over a STEM image. The $\sigma_{\text {image }}$ of an image with sharp peaks is larger than that of an image with dull peaks, under the same experimental condition such as probe current and detector. Thus, we evaluate the sharpness of atomic peak in an image with the $\sigma_{\text {image. }}$ We carried out this calculation for all images of the through focus series. Figure 1 shows an example, it shows standard deviations of images, plotted on the various defoci, and some STEM-ADF images obtained at different defoci. The convergence angle was $37 \mathrm{mrad}$ and the focus was shifted by $0.7 \mathrm{~nm}$ step in a through-focus series. The red rhomboids plot the values obtained by experiment, and the black solid line is fitted with a Gaussian function. The full width of half maximum (FWHM) fitted with the Gaussian function was evaluated as the depth resolution. Figure 2 shows the relationship between the obtained depth resolution and convergence angle. The black solid line plots the calculated values by the equation (1), and red rhomboids plot the values obtained by experiment. As the convergence angle increases, the depth resolution improves. And in the larger convergence angle, the difference between the calculated and experimental values becomes large. We presume that this is mainly due to chromatic aberration, that is, focus variation on different energy of electrons. Thus, we next measured through-focus series on different energy spreads, which were measured with EELS. 
To change the energy spread, we have changed the extraction voltage of electrode located just below an emitter in a gun chamber. When the extraction voltage is lowered, the beam currents decrease. We compensated this degrease of the beam current and adjusted it to be constant by changing excitations of condenser lenses above the condenser aperture to keep convergence angle same. In this experiment, the finally beam currents was tuned to be $30 \mathrm{pA}$ at each energy spread, and convergence angle was tuned to be $54 \mathrm{mrad}$. Figure 3 shows the relationship between the depth resolutions depending on energy spreads. The plot indicates that the depth resolution improves as the energy spread lowered. And it will extrapolate to the calculated value at zero energy spread. Thus, it was found experimentally that not only high convergence angle is required, but also chromatic aberration needs to be improved to improve the depth resolution.

References:

[1] S Morishita et al., Microscopy 67 (2018), p. 46.

[2] K Benthem et al., Ultramicroscopy 106 (2006), p. 1062.

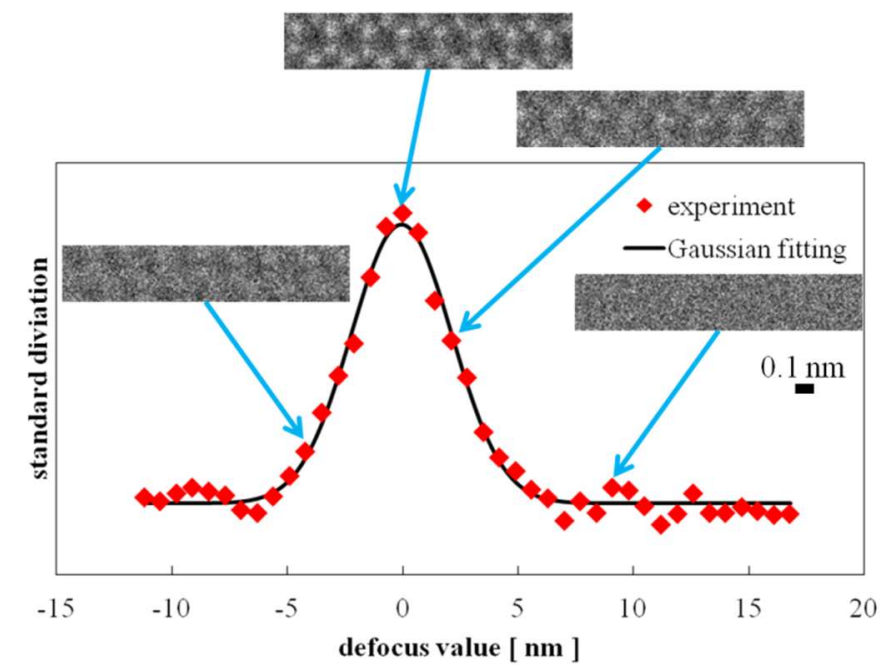

Figure 1. Standard deviations of image intensities for images obtained at different defoci plotted against the defocus and typical STEM-ADF images of mono-layer graphene obtained at corresponding defoci.

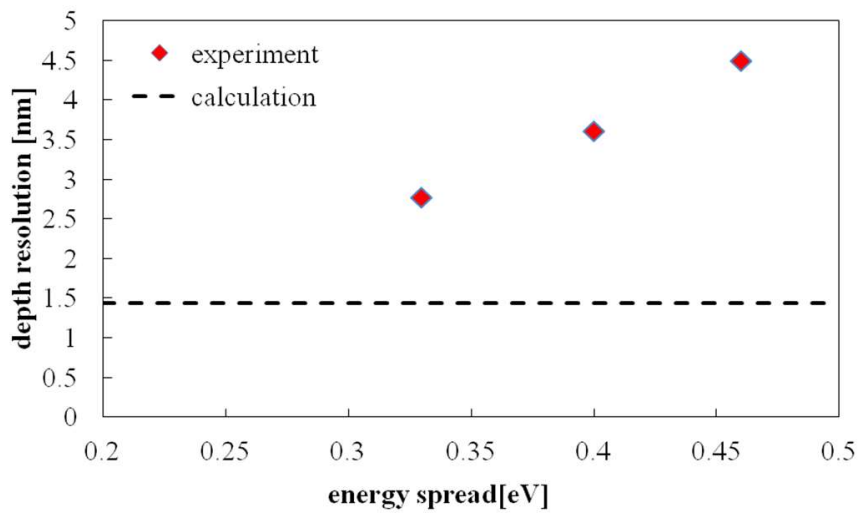

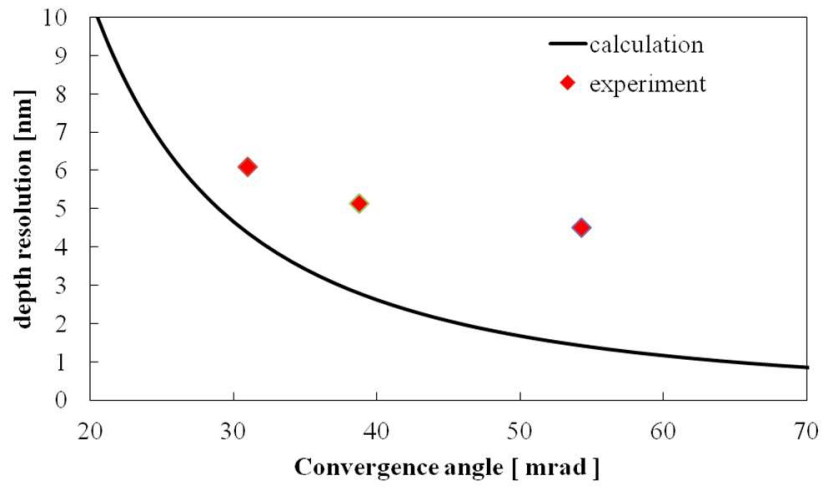

Figure 2. Relationship between depth resolution and convergence angle. The black solid line indicates calculated depth resolution on convergence angle, and red rhomboids plot experimental depth resolution.

Figure 3. Relationship between depth resolution and energy spread of the electron probe. The black dotted line indicates calculated depth resolution, and the red rhomboids plot experimental values. 